\title{
Two portable long-term measurement devices for ECG and bioimpedance
}

\author{
Timo Vuorela, Jukka Vanhala \\ Institute of Electronics \\ Tampere University of Technology \\ Tampere, Finland \\ timo.vuorela@tut.fi
}

\author{
Ville-Pekka Seppä, Jari Hyttinen \\ Ragnar Granit Institute \\ Tampere University of Technology \\ Tampere, Finland \\ ville-pekka.seppa@tut.fi
}

\begin{abstract}
This paper presents an implementation of two portable measurement device prototypes. Both prototypes are targeted for measuring ECG and bioimpedance signals. The first prototype is a wireless type, which sends data in real-time to a $\mathrm{PC}$. The second prototype is a logging device, which stores data to a memory card. After a measurement session data from memory card is transferred to a PC through USB cable. One drawback in the first prototype is a poor usability caused by the limited range of the wireless data link and another drawback is a crosstalk between measured signals. The drawbacks of the first prototype were improved in the second prototype.
\end{abstract}

Keywords: ECG, bioimpedance, portable measurement device

\section{INTRODUCTION}

ECG (Electrocardiogram) -signals are nowadays widely utilized in both clinical and consumer applications. Many wireless prototypes for measuring ECG are developed [1],[2] and e.g. many amateur athletes use commercial heart rate monitors during an exercise. ECG is a very useful physiological signal and it is also quite easy to measure. Even through many physiological parameters, such as energy consumption, can be calculated from ECG, these calculations are always based on mathematical models which are not accurate for every possible user. If more accurate results are needed other measurement techniques must be applied.

One possible candidate for a new measurement technique is a bioimpedance measurement. Bioimpedance of a tissue is measured in a same way than the conventional impedance. A small alternating current is supplied to the tissue and the alternating voltage generated by this excitation current is measured. The absolute value and phase angle of the bioimpedance is then calculated from current and voltage signals.

Just like from ECG many physiological parameters can be calculated from bioimpedance signal as well. For example different kind of body composition values e.g. amount of body fat, fat free mass in the body or body's fluid balance can be calculated. Furthermore with different measurement and electrode setup also some cardiovascular and respiration related signals can be measured.

Portable devices for measuring bioimpedance have been developed [3, 4]. Many times these devices measure only

PERVASIVEHEALTH 2008, 30 Jan - 1 Feb. Tampere, Finland

Copyright ( $)$ 2008 ICST 978-963-9799-15-8

DOI 10.4108/ICST.PERVASIVEHEALTH2008.2512 bioimpedance and are targeted for measuring only one physiological quantity e.g. TBW (Total Body Water). A measurement device capable to measure both the bioimpedance and ECG could produce information about the correlation of these two signals. Furthermore in order to perform measurements during people's normal daily activities or during sporting the measurement device should be portable and as unnoticeable as possible.

Target of this research is to develop a portable measurement device described in previous paragraph. So far two prototypes of the devices have been implemented and are described in this paper.

Rest of this paper is organized as follows; section two presents some basics of the bioimpedance measurement. Sections three and four present the implemented prototypes. In section five measurement results achieved from implemented prototypes are presented. Finally sections six and seven draw some conclusion and present future work.

\section{BIOIMPEDANCE BASICS}

Bioimpedance can be measured with two, three or four electrodes. In two and three electrode setups the impedance of the electrodes can affect to the accuracy of the measurement. Therefore, in the implemented measurement device a tetrapolar, four electrodes, measurement method is utilized. [5] In four electrode measurement two electrodes are supplying the excitation current to the tissue and other two electrodes are measuring the generated voltage. If the input impedance of the measurement amplifier is high enough the impedance of electrodes shouldn't affect the measurement. Furthermore with four electrode setup it is easier to control the actual measurement zone. Measurement principle is presented in the figure one.

In order to simplify the electronic design, the measurement devices measure only the dynamic changes in the absolute value of the bioimpedance. Measuring of both the absolute value and the phase angle accurately, would make electronics more complicated. This is due to the additional blocks needed for detecting the difference in phase between current and voltage signals, and for calibrating the devices e.g. for different temperatures. 


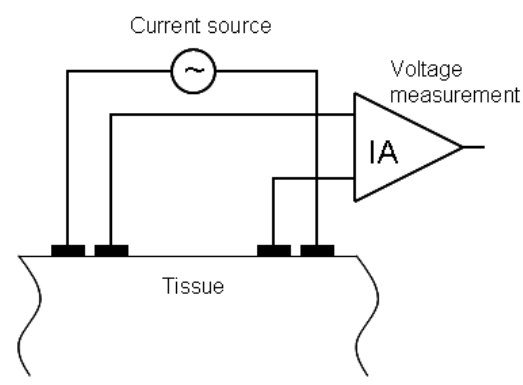

Figure 1. Principle of the tetrapolar bioimpedance measurement

\section{THE FIRST MEASUREMENT DEVICE PROTOTYPE}

The first prototype implemented in this project is presented in [6]. The operating principle of this prototype is to perform measurements and to send the data through an RF data link to a $\mathrm{PC}$, which prints measured data to a display almost in real time and stores data to a memory. Block diagram of the measurement device is presented in the figure two. The portable part of the first prototype weights 66 grams and its dimensions are $44.5 \times 63.5 \times 25 \mathrm{~mm}$. Module in question is presented in the figure three.

\section{A. Benefits and drawbacks in the first prototype}

The first prototype of the measurement prototype works rather well. Because it is battery powered and because the data is transmitted wirelessly, there is no galvanic connection to a $\mathrm{PC}$ or to the power distribution network. Therefore the signal to noise ratio (SNR) of the measured signals is high and there is hardly any $50 \mathrm{~Hz}$ interference in the signals. Power consumption of the device is $31 \mathrm{~mA}$ and with a $750 \mathrm{mAh}$ cell phone battery it can in theory operate continuously 24 hours.

Data is transferred from measurement device to a PC through an $\mathrm{ANT}^{\mathrm{TM}}$ radio link. ANT'M technology is chosen because it consumes less power than other popular technologies like Bluetooth ${ }^{\circledR}$ or ZigBee $\AA$. However the range of the radio link in this prototype is relatively short, only about ten meters. This reduces the device's usability, because the user cannot be farther than ten meters away from the base station. E.g. in the football game this range is too short. The data rate of the ANT® radio is quite low, only $20 \mathrm{kbit} / \mathrm{s}$. Due to required protocol, the amount of actual payload is only $250 \mathrm{ADC}$ samples per seconds. This means that sample rate for both channels is only little over $100 \mathrm{~Hz}$. For ECG this is enough, but bioimpedance could benefit from higher sampling rates.

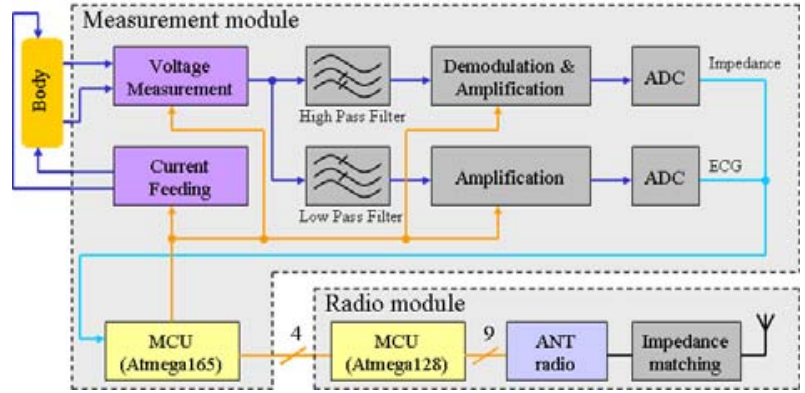

Figure 2. Block diagram of the first prototype

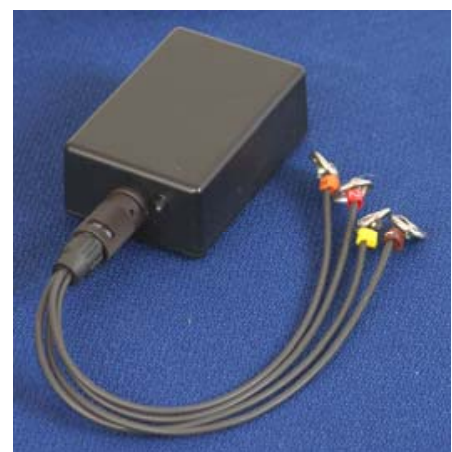

Figure 3. Portable part of the first prototype

Another drawback in the first prototype is the utilization of only one instrumentation amplifier for measuring the voltage of both the ECG and bioimpedance signals. Even though there is some filtering prior to amplification blocks the ECG is a little bit visible in the bioimpedance signal. Also some problems with the device software were detected. Software was not able to synchronize ECG and bioimpedance signals, which makes interpretation of measurement results difficult.

As can be seen form figure two there is some hardware overhead in the first prototype. Due to tight implementation schedule a two micro-processor approach was chosen. This made it possible to develop both the measurement module and radio module in parallel, which decreased the time required for implementation. However, this increases the current consumption.

\section{THE SECOND MEASUREMENT DEVICE PROTOTYPE}

The block diagram of the second measurement device prototype is presented in the figure four. The operating principle of this measurement device differs from the first prototype. Because the range of the radio link is short, the radio link was rejected in this prototype. Link is replaced with a MMC (Multi Media Card). During a measurement session device stores data to the memory card. After the measurement session the stored data is transferred to a PC through a serial cable. As can be seen from figure four this prototype contains only one microprocessor, which handles both the controlling of analogue measurements and storing the measurement samples to the MMC. Implementation of analogue filtering and amplification blocks in this prototype is identical to implementation in the first prototype and more information about these blocks can be found from [6].

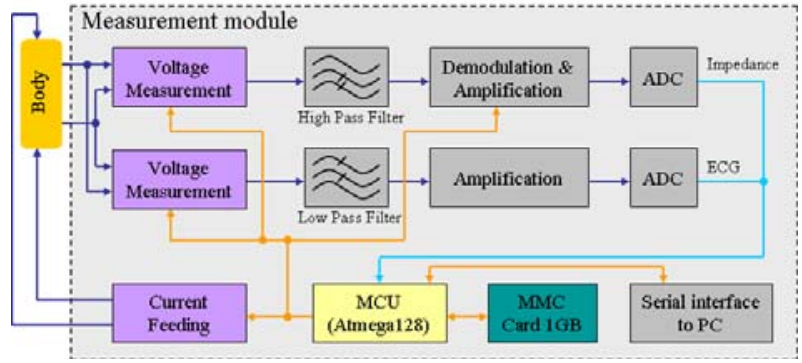

Figure 4. Blockdiagram of the second prototype 


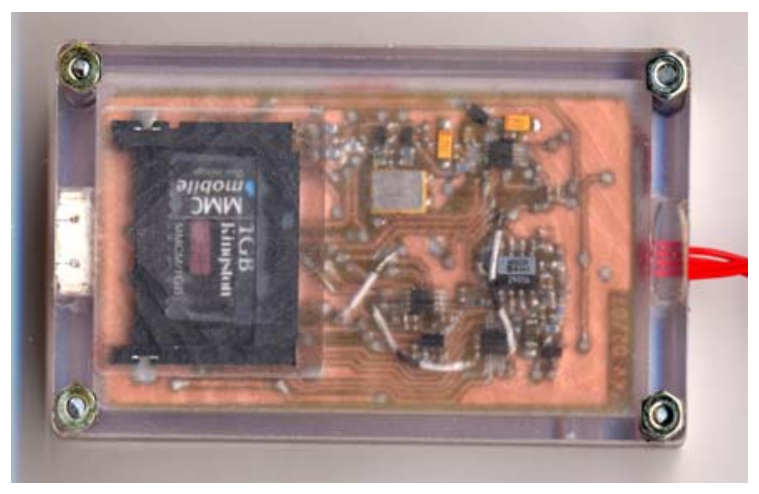

Figure 5. Second prototype

Crosstalk of bioimpedance and ECG, which was problem in the first prototype, has been decreased in the second prototype by adding separate voltage measurement instrumentation amplifiers for both signals. Voltage measurements block of ECG-measurement also contains a low pass filtering, which corner frequency is $340 \mathrm{~Hz}$. This filter removes the high frequency bioimpedance signal from ECG signal. In order to remove the low frequency ECG signal from bioimpedance signal an analogue high pass filter is added to bioimpedance voltage measurement block. Corner frequency of this filter is higher than the frequency content of the ECG signal. With this filtering arrangement the crosstalk between signals is reduced to an acceptable level.

The second prototype is encapsulated into a custom made enclosure, which is cut out from acrylic plastic. This second prototype is presented in the figure five. Dimensions of the casing are $79 \times 45 \times 20 \mathrm{~mm}$ and the weight is 84 grams. Current consumption of the measurement device is between 40 $\mathrm{mA}$ and $50 \mathrm{~mA}$, depending on sample rate. Higher current consumption than in the first prototype is caused by the memory card. Utilization of newer memory cards, e.g. SD (Secure Digital) or microSD, might decrease the current consumption. However with a $700 \mathrm{mAh}$ battery, this device has still an operating time of at least 14 hours.

Power supply of the second measurement device is a same kind of cell phone battery as in the first prototype. Now the capability to charge the battery inside the measurement device is also added. In order to make recharging as simple as possible it is performed through a computer USB (Universal Serial Bus) port. This same connection is also utilized for transferring the measured data to the PC, so battery is a little bit recharged every time the device is connected to $\mathrm{PC}$ for accessing the measurement results. Anyhow, due to the limited current available from the USB port the charging current is limited to $100 \mathrm{~mA}$. With this current charging of an empty $700 \mathrm{mAh}$ battery requires time of seven hours. Even through this is relatively long a time, device can be connected to a computer for example for overnight and in the morning battery is full.

The software of the second prototype is also improved when compared to the first prototype. This version of the software is able to synchronize the two measurement signals with each other. This helps in studying the correlation between ECG and bioimpedance signals. Because measured data is now stored into the memory card, higher sample rates than in the first device can be used. The maximum sample rate tested is $250 \mathrm{~Hz}$ per measurement channel. Even higher sample rates are possible but current software doesn't allow their utilization.

\section{MEASUREMENTS FROM PROTOTYPES}

Test measurements have been done with both measurement devices. Tests have been performed with commercial gel paste $\mathrm{Ag} / \mathrm{AgCl}$ electrodes. Figures six, seven, and eight present example measurements performed with measurement devices.

Figure six presents signals measured with the first measurement device. During the test person to be measured was sitting in the chair and electrodes were placed in horizontal line on the left side of the torso so that the distance between voltage measurement electrodes was about $20 \mathrm{~cm}$. From this electrode position respiration caused changes in bioimpedance should be relatively big. Two high peaks in bioimpedance signal are caused by changes in the lung volume. Smaller changes in the bioimpedance are caused by changes caused by heart activity. It must be noted that because of the synchronization error in the first prototype ECG and bioimpedance signals are not exactly in same phase. However very small peaks caused by the crosstalk of ECG signal are also visible in the bioimpedance signal. More measurements performed with the first prototype are presented in [7].

The measurement presented in figure seven is performed with the second measurement device. During this measurement the test person was sitting in the chair. Electrodes were placed in vertical line on the left side of the torso so that the distance between outermost, current carrying, electrodes was about 25 $\mathrm{cm}$ and the distance between voltage measuring electrodes was about $15 \mathrm{~cm}$. This kind of electrode setup is a good choice for measuring the signals caused by the heart and it can be seen from figure seven that the measured ECG-signal is quite high in amplitude. Upper signal in the figure seven presents the measured bioimpedance signal and the changes in this signal are caused by the changes in the lung volume caused by respiration activity. Small fluctuation in the presented ECG is caused by the respiration, which can be seen when the DClevel of the ECG signal is compared to the bioimpedance signal. In the figure seven signals are synchronized and it can be seen that there is no visible crosstalk between ECG and bioimpedance.

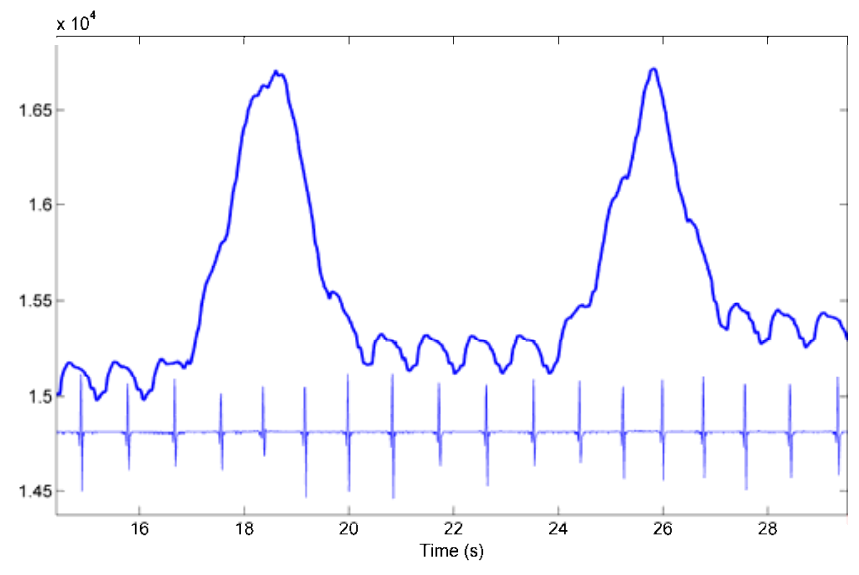

Figure 6. Bioimpedance and ECG Signals measured with the first prototype 


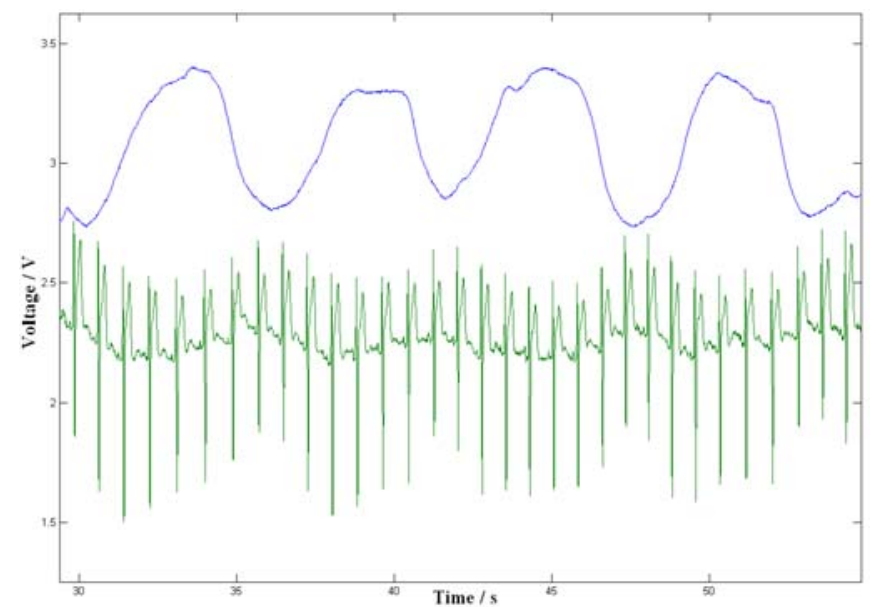

Figure 7. Bioimpedance and ECG Signals measured with the second prototype

Figure eight presents another measurement example performed with the second prototype. In this test the test person was also sitting in the chair and electrode configuration was a vertical line on the left side of the torso. The measured data is further processed with Matlab $\AA$ so that the heart rate is calculated from ECG signal. When comparing the bioimpedance signal and heart rate signal a clear correlation between these two can be seen.

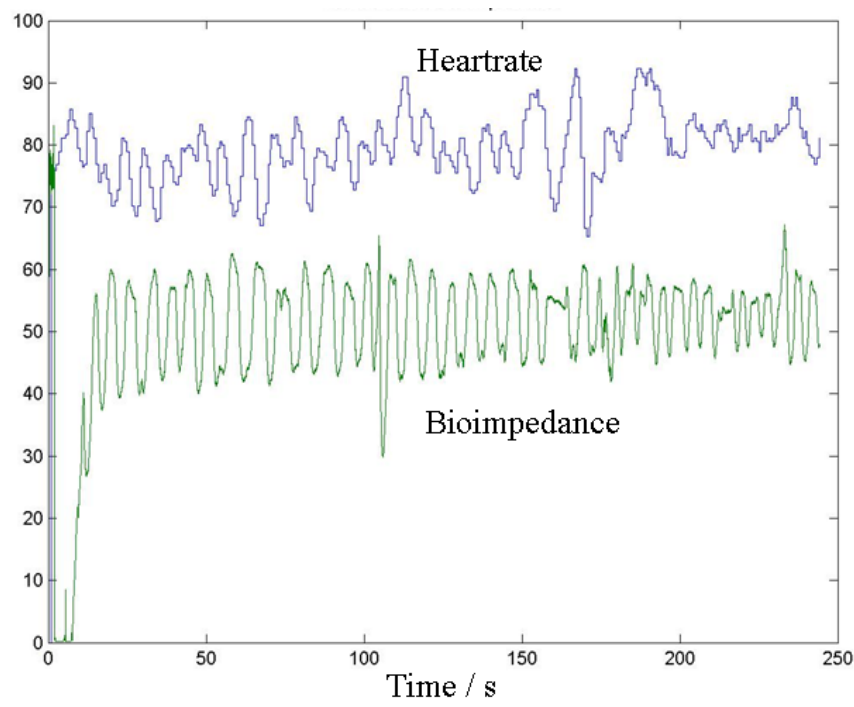

Figure 8. Bioimpedance signal (lower) measured with the second prototype and the heart rate (upper) calculated with Matlab ${ }^{\circledR}$ from measured ECG signal.

\section{CONCLUSIONS}

Two implemented measurement devices for measuring ECG and bioimpedance signals have been presented. The experienced drawbacks in operation and usability of the first prototype have been overcome in the second prototype. Both prototypes are relatively light in weight and have long operating hours with a commercial cell phone battery. Therefore it is possible to use them in long-term measurements e.g. during sporting.

\section{FUTURE WORK}

Future work includes implementation of the third prototype version. This prototype is going to be smaller in size than two prototypes presented in this paper. The plan is to encapsulate the third prototype with layers of epoxy and silicone. With these coating methods we are going to achieve a fully waterproof prototype, which could be worn e.g. during a shower after a sporting exercise. The third prototype is very much likely to utilize custom made textile electrodes instead of commercial gel-paste electrodes.

\section{ACKNOWLEDGMENT}

This research is funded by Finnish Agency for Technology and Innovation, TEKES.

\section{REFERENCES}

[1] Antti Vehkaoja, Jarmo Verho, and Jukka Lekkala, Miniature Wireless Measurement Node for ECG Signal Transmission in Home Area Network, Proceedings of the $28^{\text {th }}$ IEEE Annual International Conference on Engineering in Medicine and Biology Society EMBC 2006, 30 Aug 3 Sept, New York City, USA, pp. $2049-2052$.

[2] M. Karlsson, et al., Wireless System for Real-Time Recording of Heart Rate Variability for Home Nursing, Proceedings of the $27^{\text {th }}$ IEEE Annual Conference on Engineering in Medicine and Biology Society EMBC 2005, 1 - 4 Sept, Shanghai, China, pp. 3717 - 3719.

[3] Timo Vuorela, Kari Kukkonen, Jaana Rantanen, Tiina Järvinen, nad Jukka Vanhala, Bioimpedance Measurement System for Smart Clothing. Proceedings of the Seventh International Symposium on Wearable Computers ISWC 2003, 21-23 October, White Plains, New York, USA. pp. $98-107$.

[4] E. Calderón, A. Guimerà, P. Los, A. Mouroux, N. Pitts, Multichannel Handheld Hard-tissue Bioimpedance Meter with Bluetooth Link, Proceedings of the $13^{\text {th }}$ International Confrence on Electrical Bioimpedance, ICEBI 2007, $29^{\text {th }}$ Aug - $2^{\text {nd }}$ Sept, Graz, Austria, pp. 244-247.

[5] S. Grimnes, and Ø. G. Martinsen, Bioimpedance \& Bioelectricity Basic, Academic Press, London, 2000.

[6] Timo Vuorela, Ville-Pekka Seppä, Jukka Vanhala, and Jari Hyttinen, Wireless Measurement System for Bioimpedance and ECG, Proceedings of the $13^{\text {th }}$ International Confrence on Electrical Bioimpedance, ICEBI 2007, $29^{\text {th }}$ Aug - $2^{\text {nd }}$ Sept, Graz, Austria, pp. 248-251.

[7] Measuring Respirational Parameters with a Wearable Bioimpedance Device, Ville-Pekka Seppä, Juho Väisänen, Pasi Kauppinen, Jaakko Malmivuo, and Jari Hyttinen, Proceedings of the $13^{\text {th }}$ International Confrence on Electrical Bioimpedance, ICEBI 2007，29 $9^{\text {th }}$ Aug - $2^{\text {nd }}$ Sept, Graz, Austria, pp. 663-666. 